\title{
Validación española del «intention to partake in leisure-time physical activity» Spanish validation of the scale «intention to leisure-time in partake physical activity»
}

\author{
Antonio Granero-Gallegos*, Antonio Baena-Extremera**, Francisco Javier Pérez-Quero***, María del Mar Ortiz-

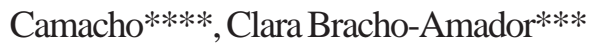 \\ *Junta de Andalucía, **Universidad de Murcia, ***Universidad de Huelva, ****Universidad de Granada
}

Resumen: El objetivo de esta investigación ha sido validar al español la escala Intention to partake in leisure-time physical activity mediante la realización de dos estudios independientes. En el primero se utilizó una muestra piloto de 224 alumnos de educación secundaria de 12 a 19 años y se realizó una exploración de la estructura dimensional de la prueba. En el segundo estudio se ha utilizado la totalidad de la muestra con 856 alumnos de educación secundaria de 12 a 19 años, analizándose la estructura del instrumento mediante procedimientos confirmatorios. La versión española de la escala mostró niveles aceptables de consistencia interna y estabilidad temporal. La validez de constructo se exploró mediante correlación con otras dimensiones y diferencias por sexo y edad. Las correlaciones fueron positivas con la satisfacción, importancia de la Educación Física y motivación intrínseca, y negativa con aburrimiento. Asimismo, se obtuvieron diferencias significativas tanto por sexo como por edad.

Palabras clave: intención-PFTL; Educación Física; tiempo libre; propiedades psicométricas.

\begin{abstract}
The objective of this research was to validate to Spanish the scale Intention to partake in leisure-time physical activity by performing two independent studies. In the first one, a pilot sample of 224 secondary school students aged 12 to 19 years was used and it was performed a scan of the dimensional structure of the test. In the second study we used the whole sample with 856 high school students from 12 to 19 years, analyzing the structure of the instrument through confirmatory procedures. The Spanish version of the scale showed acceptable levels of internal consistency and temporal stability. Construct validity was explored by correlation with other dimensions and differences by sex and age. The correlations were positive with satisfaction, the importance of Physical Education and intrinsic motivation, and negatively with boredom. Furthermore, significant differences were obtained by sex and age.
\end{abstract}

Key words: intention-PFTL; Physical Education; leisure-time; psychometric properties.

\section{Introducción}

Dada la importancia que tiene la práctica de ejercicio físico en la salud y en la calidad de vida de las personas, se ha sugerido que un estilo de vida activo en la edad adulta puede tener su origen en un estilo de vida activo en la adolescencia (Gómez-López, Granero-Gallegos \& BaenaExtremera, 2010). Por ello, laEducación Física(EF)escolar debepromover y fomentar la práctica de actividad física por parte del alumnado más allá de los propios límites de los planes de estudios, como ocupación del tiempo libre (Taylor, Ntoumanis, Standage \& Spray, 2010).

Sobre esto, diversas investigaciones han estudiado los motivos y las intenciones por las cuales los estudiantes realizan prácticas deportivas en su tiempo de ocio (Gómez-López, Granero-Gallegos, BaenaExtremera \& Ruiz-Juan, 2011; Standage, Duda \& Ntoumanis, 2003), comovariableintencional de realizar ejercicio físico en el futuro (Lintunen, Valkonen, Leskinen \& Biddle, 1999; Ntoumanis, 2001).

En relación a las motivaciones, una aproximación teórica de esta situación puede describirse desde la Teoría de la Autodeterminación (Deci \& Ryan, 1985, 1991). Esta teoría se entiende como un continuo en el que se establecen diferentes niveles de autodeterminación. Es decir, de mayor a menor grado de autodeterminación, la conducta del sujeto puede estar intrínsecamentemotivada (realizando las prácticas deportivas por el placer y disfrute), extrínsecamente motivada o amotivada (Deci \& Ryan, 1985, 2000).

Otra perspectiva más cercana a esta investigación proviene desde las intenciones y no desde las motivaciones. Por ello, desdelaperspectiva de las teorías socio-cognitivas contemporáneas más populares en psicología de la salud, se considera la intención como el mayor determinante en las acciones futuras de ejercicio físico (Ajzen, 1985; Ajzen \& Fishbein, 1980; Ajzen \& Madden, 1986; Bandura, 1977).

Uno de los modelos teóricos que explican el comportamiento y las intenciones es la Teoría de laAcción Razonada(TRA)(Ajzen \& Fishbein, 1980). En esta Teoría, la intención puede predecir el comportamiento

Fecha recepción: 26-01-14- Fecha envío revisores: 26-01-14- Fecha de aceptación: 26-02-14 Antonio Baena Extremera

C/ Argentina s/n

30720 Santiago de la Ribera (Murcia)

abaenaextrem@um.es real dado por varios motivos; en primer lugar, porque la conducta se encuentra bajo el control total voluntario; en segundo lugar, porque la intención no cambia antes de que el comportamiento sea observado; y tercero, porque las intenciones se corresponden con un comportamiento en relación a los objetivos y al contexto en el que se encuentre el sujeto.

Otra de las Teorías explicativas de las intenciones es la Teoría del Comportamiento Planificado (TPB) (Azjen, 1985, 1991), que expone que el comportamiento no está bajo control completamente voluntario cuando éste se ve impedido por factores personales y/ o medioambientales. Esta teoría intenta predecir los comportamientos que no son completamente voluntarios por la incorporación de las percepciones de control sobre la ejecución de la conducta, como predictor adicional de la intención y el comportamiento (Ajzen, 1991). Así, la TPB sugiere que el determinante en el comportamiento voluntario es nuestra intención de participar en ese comportamiento (Rhodes, Courneya \& Jones, 2004).

A partir de estas teorías, diversos instrumentos han sido utilizados para evaluar las motivaciones e intenciones de prácticas, destacando entre ellos el Intentions to partake exercise in leisure time. Este instrumento, utilizado por Chatzisarantis, Biddle, y Meek (1997), está basado en Ajzen y Madden (1986), y mide la intención de hacer ejercicio en el tiempo libre a través de tres preguntas: 1) Tengo la intención de hacer ejercicio físico...; 2) Tengo planeado hacer ejercicio físico...;3) Estoy decidido a hacer ejercicio físico.... Las intenciones de realizar ejercicio en el tiempo libre se miden preguntando a los alumnos sobre sus intenciones, diferenciando de si «tienen que» o si realmente «quieren» hacer ejercicio físico. Por lo tanto, haciendo referencia a las teorías anteriores, los valores altos se integran en intenciones que representan experiencias autónomas, mientras que las puntuaciones más bajas, se integran en intenciones que se experimentan de forma controlada (Deci \& Ryan, 1985).

Taylor et al.(2010) utilizaron esta escala en una población estudiantil de 11 a 16 años, concluyendo que las intenciones de ejercicio físico en el tiempo libre aumentaban en esta población si el profesor de EF satisfacía sus necesidades psicológicas y conseguía en ellos una mayor motivación autodeterminada. Estos mismos datos, se corroboraban en el trabajo de Cox, Smithy Williams (2008), quienes obtuvieron los mismos resultados, 
relacionándose perfectamente la motivación intrínseca con la intención de práctica en el tiempo libre.

Otros autores, como Goudas, Biddle y Fox (1994), encontraron asociación entre las experiencias autónomas de los niños en clases de EF con las intenciones de practicar ejercicio físico en su tiempo de ocio. $\mathrm{O}$ por ejemplo Papaioannou (2000), quien encontró relación entre el comportamiento y las intenciones de práctica de ejercicio físico en estudiantes de 6 a 14 años.

Moreno y Hellín (2002) encontraron, en una muestra de secundaria, que los alumnos prefieren la EF a otras asignaturas y hacer EF y deporte a realizar otras actividades, disminuyendo esta tendencia conforme aumentan de edad. Igualmente, Ntoumanis, Barkoukis y ThøgersenNtoumani (2009) afirman que la motivación de los alumnos hacia la EF y hacia la actividad física comienza a disminuir en los adolescentes conforme avanzan en edad. De hecho, la motivación de los alumnos hacia esta área no es síntoma de que los discentes practiquen actividad física fuera del ámbito escolar. En consonancia con esto, Román, Serra, Ribas, Pérez-Rodrigo y Aranceta (2006) hallaron que cerca del 70\% de los niños y adolescentes españoles no realizan actividad física regular en su tiempo libre, especialmente las chicas, siendo estos datos muy diferentes a los del resto de países europeos. Por ello, es fundamental acometer esta investigación, donde el objetivo principal es validar al idioma y al contexto español, la escala de Intention to partake in leisuretime physical activity, para poder hacer mediciones y comparaciones con la población de otros países y con la nuestra propia.

En el presente trabajo se presentan los resultados de dos estudios independientes y se expone el proceso traducción y adaptación de los ítems, así como los datos relacionados con las evidencias de validez de contenido delos mismos. En primer estudio se realizó con la participación de 224 alumnos de educación secundaria. Tras exponer el proceso de adaptación de losítems, y mostrados los datos relacionados con evidencias de validez de contenido, se llevó a cabo un análisis estadístico de los datos, una exploración de la estructura dimensional de la prueba, y un análisis dela fiabilidad del instrumento. En el segundo estudio participaron un total de 856 estudiantes de educación secundaria y se analiza, mediante modelos de ecuaciones estructurales, la estructura factorial confirmatoria del instrumento, a la vez que se pone a prueba otra vez la consistencia interna, estabilidad temporal y validez concurrente. Para la elaboración de este trabajo se siguieron las directrices propuestas por CarreteroDios y Pérez (2007) para los estudios instrumentales y que han sido utilizadas en la validación de la versión española de diversas escalas psicológicas de aplicación al ámbito educativo (Baena-Extremera, Granero-Gallegos, Bracho-Amador \& Pérez-Quero, 2012).

Estudio 1: Estudio psicométrico preliminar de la escala "Intention to partake in leisure-time physical activity"

\section{Material y método}

\section{Participantes}

En este primer estudio participaron un total de 224 alumnos (110 varones $=49.1 \%$; 114 mujeres $=50.9 \%$ ) de dos centros de Educación Secundaria de la provincia de Almería. El rango de edad estuvo comprendido entre 12 y 19 años $(M=14.68 ; D T=1.27)$, siendo la edad media de los chicos $14.90(D T=1.23)$, y la de las chicas $14.47(D T=$ 1.28).

\section{Instrumento}

Intention to partake in leisure-time physical activity (Chatzisarantis, et al., 1997). Este instrumento consta de una escala de tres ítems y los citados autores los redactaron sobre la base de la obra de Ajzen y Madden (1986), teniendo en cuenta que la redacción correspondiera con el criterio de comportamiento en el tiempo, el contexto, objetivo y la acción (Ajzen \& Fishbein, 1980). El instrumento trata de evaluar la intención de los estudiantes de ser físicamente activos en su tiempo libre durante el período de un mes. Para ello respondieron a tres preguntas, sobre el encabezado de «En mi tiempo libre, fuera del instituto...»: (1) Tengo intención de hacer ejercicio físico al menos tres veces por semana en el próximo mes; (2) Tengo planeado hacer ejercicio físico al menos tres veces por semana en el próximo mes; (3) Estoy decidido a hacer ejercicio físico al menos tres veces por semana en el próximo mes. Las respuestas se recogen en una escala tipo Likert de 7 puntos que van de 1 (muy improbable) a 7 (muy probable). Los coeficientes alfa de Cronbach (á) de consistencia interna obtenida por Chatzisarantis et al. (1997) fue de .89 y .90 para las intenciones de pre y post ejercicio, respectivamente. Similares coeficientes de consistencia interna (alfa = .89) hallaron otros autores como Standage et al. (2003) en estudiantes de secundaria. La consistencia interna en este trabajo fue .91.

Traducción y fases previas del proceso de adaptación del instrumento al español

La adaptación española de la escala Intention to partake in leisuretime physical activity se realizóatendiendo a los estándares metodológicos internacionales recomendados por la International Test Comision(ITC) para adaptar correctamente test y escalas de unas culturas a otras (Hambleton, 2005; Muñiz \& Bartram, 2007). Para evitar imprecisiones, se combinaron los diseños de traducción directa e inversa de los ítems, según el procedimiento parallel backtranslation (Brislin, 1986).

La evaluación cualitativa deítems (validez de contenido) se efectuó mediante el juicio de cuatro expertos (Osterlind, 1989): dos en construcción de escalas y dos conocedores del constructo a evaluar. Todos los ítems fueron analizados y revisados se llegó a hasta que se recogiese la dimensión teórica de la forma más clara y precisa.

La nueva versión fue administrada a 50 alumnos de secundaria con edades entre 12 y 19 años. Los comentarios de los mismos sobre instrucciones y forma de redacción supusieron cambios menores. Tras el análisis de los resultados psicométricos obtenidos yunaúltima revisión por parte del equipo de investigación se llegó a la versión final española del Intention to partake in leisure-time physical activity (Intención-PFTL) (Anexo).

\section{Procedimiento}

Se obtuvo autorización para realizar la investigación por parte de los órganos de dirección de los centros educativos. Los estudiantes fueron informados del propósito del estudio y de sus derechos como participantes en el mismo. Los instrumentos para medir las diferentes variables se administraron en el aula, no estando presente el docente. Cada participante tuvo 10-20 minutos para completar los cuestionarios. Las respuestas al instrumento se mantendrán en el anonimato.

\section{Análisis estadísticos de los datos}

Se llevó a cabo un análisis de ítems y homogeneidad de la escala. Se realizó un análisis factorial exploratorio (EFA) para obtener la estructura factorial interna y se analizó la consistencia interna con el objeto de determinar la fiabilidad(alfa) de Cronbach. Se utilizó el SPSS 17.0.

\section{Resultados}

\section{Análisis de la estructura interna}

Para conocer la estructura factorial empírica de la versión española de la escala Intención de practicar actividad física en el tiempo libre se llevó a cabo un análisis factorial exploratorio sobre los 3 ítems de la escala. Se utilizó el método de extracción de componentes principales, considerando valores propios $>1$ y requiriéndose una correlación mínima de .40 para que cada ítem sea importante dentro del factor (Stevens, 1992). Como recomiendan Bollen y Long (1994), los índices de asimetría y curtosis son próximos al valor $0 \mathrm{y}<2$ (Tabla 2).

Atendiendo a las recomendaciones de Carretero-Dios y Pérez(2007), es necesario presentar, antes de la aplicación del análisis, los estimados que aseguren que la matriz de correlaciones es apropiada, recomendándose el cálculo de la prueba de esfericidad de Bartlett y el índice Kaiser-Meyer-Olkin (KMO) de adecuación muestral. El índice KMO mostró valores aceptables y el test de Bartlett resultó estadísticamente significativo (Tabla 1), lo que permitió concluir que la aplicación del EFA resultaba pertinente. 
Con un porcentaje total de varianza explicada del $87.87 \%$, los resultados del EFA ponen de manifiesto la estructura dimensional de la escala en un factor con saturaciones de los ítems por encima de .90, ajustándose al constructo teórico inicial propuesto. El factor se denominó Intención-PFTL (Intención de práctica física en tiempo libre).

\section{Análisis de ítems y homogeneidad de la escala}

El procedimiento de análisis se realizó en función de lo establecido por Carretero-Dios y Pérez (2007). Se llevó a cabo el análisis estadístico de los ítems de la escala. Los criterios para conservar un ítem fueron: valor e».30 en el coeficiente de correlación corregido ítem-total (CCIT$c$ ), desviación típica $(D T)>1$, y corroborar que todas las opciones de respuesta habían sido usadas en algún momento (Nunnally \& Bernstein, 1995).

La escala presenta unos valores entre 5.17 y 5.35, como se puede comprobar en la Tabla 2. Los tres ítems presentaron $D T>1$. Todos los elementos presentaron una correlación ítem-total dentro de los límites aconsejables, siendo el más bajo $r=.83$. La consistencia interna de la escala fue adecuada (alfa = .93) y la eliminación de alguno de los ítems no supondría un incremento de la fiabilidad.

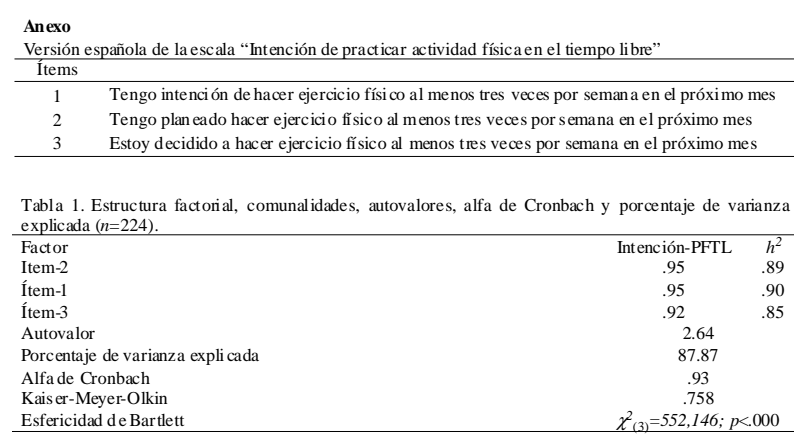

Tabla 2. Estadísticos descriptivos, de consistencia intema y de homogeneidad $(n=224)$.

\begin{tabular}{ccccccc}
\hline Escala: Intención-PFTL (al fa =.93) & M & DT & CCIT-c & $\begin{array}{c}\text { alfa sin } \\
\text { item }\end{array}$ & Asimetría & Curtosis \\
\hline Ítem 2 & 5.17 & 1.91 & .87 & .88 & -.81 & -.52 \\
Ítem 3 & 5.28 & 1.91 & .83 & .92 & -.89 & -.37 \\
Ítem 1 & 5.35 & 1.84 & .87 & .88 & -.92 & -.20
\end{tabular}

Estudio 2: Análisis factorial confirmatorio, validez de constructo, consistencia interna y estabilidad temporal

El objetivo de este trabajo es aportar evidencias sobre la dimensionalidad de la versión española de la escala «Intention to partake in leisure-time physical activity» en una muestra de adolescentes estudiantes de educación secundaria aplicando procedimientos confirmatorios. Se realizó un análisis de las propiedades psicométricas de la citada escala; para ello se concretó: (a) examinar su estructura factorial con análisis factorial confirmatorio (AFC), (b) evaluar la consistencia interna mediante alfa de Cronbach, coeficiente de fiabilidad compuesta y varianza media extractada, (c) verificar su estabilidad temporal, y (d) evaluar las diferencias de sexo y edad a partir de las medias obtenidas.

\section{Material y método \\ Participantes}

En este estudio participaron un total de 856 alumnos (415 hombres = 48.5\%; 441 mujeres $=51.5 \%$ ) de Educación Secundaria de las provincias de Almería, Córdoba, Granada, Jaén, Málaga y Sevilla. El rango de edad estuvo comprendido entre 12 y 19 años $(M=15.02$; $D T$ $=1.44)$, siendo la edad media de los chicos $15.07(D T=1.42)$, y la de las chicas $14.95(D T=1.46)$.

Para evaluar la estabilidad temporal de la versión española de la escala «Intention to partake in leisure-time physical activity» se utilizó una segunda muestra de 187 alumnos, elegida previamente de forma aleatoria y compuesta por 93 chicos $(M=15.02 ; D T=1.37)$ y 94 chicas $(M=14.95 ; D T=1.42)$ que completaron nuevamente el instrumento siete semanas más tarde. Con el objeto de prevenir posibles tendencias de deseabilidad social los adolescentes fueron instruidos para queutilizaran su fecha de nacimiento para identificar sus cuestionarios, al igual que se ha realizado en estudios recientes (Baena-Extremera, et al., 2012).

\section{Instrumentos}

Fue administrada la versión española de la escala «Intention to partake in leisure-time physical activity», derivada del estudio 1 . Se recabaron, además, datos socio-demográficos de los participantes.

Para analizar la validez de constructo de la versión en castellano de la anterior se escogieron tres subescalas que teóricamente debían correlacionar positivamente con este constructo (satisfacción/diversión con la práctica deportiva, importancia de la Educación Física y motivación intrínseca) y una subescala que en teoría debía correlacionar de manera negativa (aburrimiento con la práctica deportiva).

Escala de Satisfacción(SSI). Se utilizó la versión española adaptada al contexto de la EF (SSI-EF) (Baena-Extremera, et al., 2012) del original Sport Satisfaction Instrument (Duda \& Nicholls, 1992). Consta de 8 ítems que miden la satisfacción intrínseca en las clases de EF mediante dos subescalas: satisfacción/diversión y aburrimiento. En las instrucciones se pide a los alumnos que indiquen el grado de acuerdo con losítems que reflejan criterios de diversión o aburrimiento. Las respuestas se recogieron en una escala de ítems politómicos con rango de puntuación entre 1 (muy en desacuerdo) y 5 (muy de acuerdo). Baena-Extremera et al. (2012) informaron que la versión española del SSI-EF ha demostrado propiedades psicométricas adecuadas y encontraron unos índices de fiabilidad entre alfa $=.92$ para la satisfacción/diversión y alfa $=.79$ para el aburrimiento. En esteestudio se halló un alfa $=.92$ para la satisfacción/ diversión y un alfa $=.78$ para el aburrimiento.

Importancia de la Educación Física (PEI). Instrumento original de Moreno, González-Cutre y Ruiz (2009) para medir la importancia y utilidad de la EF entre el alumnado. Consta de una escala de tresítems y se pide a los sujetos que indiquen el grado de acuerdo con los ítems que reflejan criterios de importancia de la Educación Física, recogiéndose las respuestas en una escala de ítems politómicos de 4 puntos que oscila desde totalmente en desacuerdo(1) a totalmente de acuerdo (4). Moreno et al. (2009) demostraron la validez interna de esta escala en estudios con adolescentes españoles, con valores alfa $=.75$. En este trabajo la consistencia interna de la escala fue de alfa $=.76$.

Motivación Intrínseca. Seutilizóla subescala de motivaciónintrínseca de la Escala de motivación en el deporte (SMS). Se empleó la versión validada al castellano por Núñez, Martín-Albo, Navarro \& González (2006) y adaptada a la EF de la SMS de Brière, Vallerand, Blais \& Pelletier(1995). Esta escalamidela desmotivación(4ítems), lamotivación extrínseca (12 ítems) y la motivación intrínseca (12 ítems). Se utilizaron los ítems correspondientes a la motivación intrínseca en una sola dimensión, como sugieren Guzmán, Carratalá y Carratalá (2005) y Martens y Weber (2002). Las respuestas se recogieron en una escala de ítems politómicos con un rango de puntuación entre 1 (totalmente en desacuerdo) y 7 (totalmente de acuerdo). Otros estudios que han utilizado este instrumento en estudiantes de EF (Granero-Gallegos, BaenaExtremera, Pérez-Quero, Ortiz-Camacho \& Bracho-Amador, 2012) demostraron la validez interna de la dimensión (alfa $=.93$ ). En este trabajo la consistencia interna de la escala fue de alfa $=.93$.

\section{Procedimiento}

Se obtuvo autorización para realizar la investigación por parte de los órganos de dirección de los centros educativos. Los estudiantes fueron informados del propósito del estudio y de sus derechos como participantes en el mismo. Los instrumentos para medir las diferentes variables se administraron en el aula, no estando presente el docente. Cada participante tuvo 10-20 minutos para completar los cuestionarios. Las respuestas al instrumento se mantendrán en el anonimato.

\section{Análisis estadísticos}

Para evaluar la estructura factorial de la escala se realizó un $A F C$ con LISREL 8.80. Los análisis de ítems, homogeneidad, estructura interna, correlación, consistencia interna de la escalay las pruebas para determinar las diferencias según sexo y edad se realizaron con el SPSS 17.0. 


\section{Resultados}

\section{Descripción inicial de los datos}

En la Tabla 3 se exponen los estadísticos descriptivos de los 3 ítems de la escala (media, desviación típica, correlación ítem-total, alpha la escala teórica y la dimensión si algún ítem es eliminado, asimetría y curtosis). Como se puede comprobar, y según las recomendaciones de Bollen y Long (1994) los índices de asimetría y curtosis son próximos a $0 \mathrm{y}<2$.

En la tabla 3 se muestran los estadísticos descriptivos del IntenciónPFTL. Como se puede observar, los estadísticos fueron similares a los del estudio 1.

\section{Análisis factorial confirmatorio}

Con el objeto estudiar las propiedades psicométricas de la dimensionalización original propuesta teóricamente por Chatzisarantis et al. (1997) se aplicaron modelos de ecuaciones estructurales. La estructura factorial del Intention to partake in leisure-time physical activity se evaluó con AFC. Este análisis se llevó a cabo utilizando el método de estimación weighted least squares (WLS) para variables ordinales del programa LISREL 8.80 (Jöreskog \& Sörbom, 1993). La matriz de correlaciones policóricas y la matriz de covarianzas asintóticas fueron utilizadas como input para el análisis de los datos. Se hipotetizó un modelo de medida de un solo factor. Al ser una escala de tres variables, el ajuste del modelo daba perfecto, por lo que, atendiendo las sugerencias de Jöreskog y Sörbom (1993), se fijó la varianza del error de una de las variables observadas.

Como recomiendan diversos autores (Bentler, 2007; Markland, 2007), para la evaluación de los modelos se calcularon varios índices de ajuste, combinando índices absolutos y relativos. Entre los absolutos se utilizó el valor $p$ asociado con el estadístico chi cuadrado $\left(\chi^{2}\right)$, que prueba el modelo nulo frente al modelo hipotetizado (Barrett, 2007). La ratio entre $\chi^{2}$ y grados de libertad $(g l)(\div 2 / g l)$ es un heurístico que se utiliza para reducir la sensibilidad del $\chi^{2}$ al tamaño de la muestra. Las ratios $<2.0$ se consideran como indicadores de muy buen ajuste del modelo (Tabachnik \& Fidell, 2007). Se ha calculado el GFI (índice de bondad de ajuste) y autores como Hooper, Coughlan, y Mullen (2008) consideran valores e».95 para un mejor ajuste. Bentler(2007)recomienda reportar el RMR (residuo cuadrático medio), debiendo ser $<.08$. Entre los índices relativos se utilizaron: NFI (índice de ajuste normalizado), NNFI (índice de ajuste no normativo) y CFI (índice de ajuste comparativo). En los índices incrementales se considera que valores e».95 indican un buen ajuste (Hu \& Bentler, 1999). Autores como Kline (2005) recomiendan la utilización de RMSEA (error de aproximación cuadrático medio) como medida de parsimonia y, según Hu y Bentler (1999) un valor d».06 indicaría un buen ajuste. Los parámetros estimados se consideran significativos cuando el valor asociado al valor tes superior a $1.96(p<.05)$.

En el modelo todos los ítems presentaron valores superiores a .075 en la fiabilidad individual $\left(R^{2}\right)$; en todos los casos las cargas factoriales

\begin{tabular}{|c|c|c|c|c|c|c|}
\hline Es cala: Intención-PFTL(alfa =.93) & M & $D T$ & CCIT-c & $\begin{array}{c}\text { alfa } \sin \\
\text { item }\end{array}$ & $\begin{array}{c}\text { Asimetrí } \\
\text { a }\end{array}$ & Curtosis \\
\hline It tem 2 & 5.03 & 1.89 & .87 & .87 & -63 & -.76 \\
\hline Ítem 3 & 5.06 & 1.94 & .83 & .86 & -66 & -.79 \\
\hline
\end{tabular}

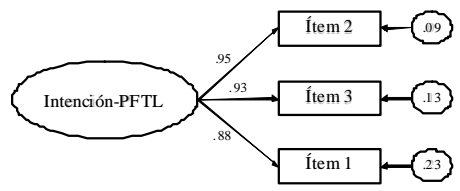

Figura 1.- Path Diagram del $A F C$, con pes os estandarizados y errores de medición de cada uno de los items del Intención-PFTL.

Tabla 4. Consistencia interna de la escala.
Coeficientes

Intención-PFTL

iabilidad Varianz
Alfa de Cronbach .93 estandarizadas fueron $>.60$ y unt-value $>1.96$ (Figura 1), lo que garantiza la validez convergente (Hair, Black, Babin \& Anderson, 2009).

Este modelo presentó unos excelentes índices de bondad de ajuste, resultando, además, el valor de $p$ significativo, como recomiendan autores como Barrett (2007), para asegurar el mejor ajuste del modelo: $\chi^{2}=1.93$, $g l=1, p=.165, \chi^{2} / g l=1.93, \mathrm{GFI}=1.00, \mathrm{RMR}=.02, \mathrm{NFI}=1.00$, $\mathrm{NNFI}=.99, \mathrm{CFI}=1.00, \mathrm{RMSEA}=.03$.

Asimismo, se considera importante en el $A F C$ de las escalas con naturaleza ordinal de la matriz de correlaciones de datos, ofrecer los datos de fiabilidad compuesta para cada una de las dimensiones críticas, pues analiza las relaciones entre las respuestas a los ítems y la variable latente medida (Elosua \& Zumbo, 2008), así como la varianza extraída para estudiar la validez de la escala.

El coeficiente de fiabilidad compuesta (Composite Reliability) se considera más adecuado que el alfa de Cronbach porque no depende del número de atributos asociados a cada concepto (Vandenbosch, 1996). Se considera que debe tomar un valor mínimo de .70 (Hair et al., 2009). En la Tabla 4 se comprueba que la fiabilidad compuesta del modelo es de .94

La varianza extraída o varianza media extractada (AVE - Average Variance Extracted), por su parte, refleja la cantidad total de la varianza de los indicadores recogida por el constructo latente. Cuanto mayor sea su valor, más representativos son los indicadores de la dimensión crítica en la que cargan. En general, se sugiere que su valor debe exceder del .50 (Hair, et al., 2009). En el modelo estudiado, $A V E=.85$.

La estabilidad temporal se evaluó con la citada muestra de 187 alumnos que completaron la escala «Intención de practicar actividad física en el tiempo libre» en dos ocasiones con intervalo de siete semanas. Tanto los resultados del pretest (alfa $=.92 ;$ ) como del postest (alfa $=$ .93;) fueron satisfactorios. Los valores de correlación test-retest en la dimensión Intención-PFTL fueron $r=.89$.

\section{Análisis de validez de constructo}

En referencia a la validez de constructo del Intención-PFTL, se realizó un análisis de correlación con otras escalas, diferenciando entre varones y mujeres. Los resultados obtenidos muestran altas correlaciones positivas con la satisfacción/diversión, la importancia y utilidad de la EF y la motivación intrínseca. Por contra, destacar la alta correlación negativa obtenida con la subescala de aburrimiento (Tabla 5).

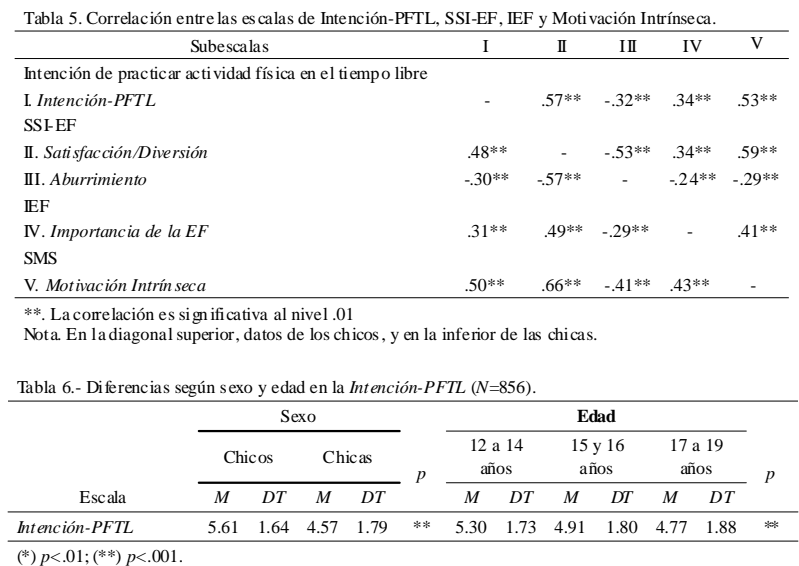

\section{Diferencias por sexoyedad}

Para analizar las diferencias según el sexo del alumnado se llevó a cabo la Prueba t de Student para muestras independientes y para estimar las diferencias según la edad se realizó un ANOVA. Se tuvo en cuenta el test de Levene, de homogeneidad de las varianzas, para estimar la igualdad de las varianza con un nivel de significación de $p<.001$. Los datos más relevantes y que hacen referencia a la $M, D T$ y nivel de significación se recogen en la Tabla 6. El t-test indicó diferencias significativas entre chicos y chicas $(F=22.98 ; p<.001)$, respecto a la intención de practicar actividad física en el tiempo libre, con datos muy por encima entre los varones. Según la edad, el ANOVA también mostró diferencias 
significativas $(F=11.04 ; p<.001)$. A medida que se avanza en edad disminuye la intención de practicar actividad física en el tiempo libre. El contraste post hoc (Bonferroni) arrojó diferencias significativas ( $p<$ .001) entre los de 12 y 14 años y el resto de grupos de edad.

\section{Discusión}

Las motivaciones e intenciones han sido estudiadas a lo largo de la historia como predictores de las acciones futuras relacionadas con la práctica de actividad física. Como afirman Cox et al. (2008), existen aún pocas investigaciones donde se estudien cómo las experiencias de la actividad física realizada durante la escuela se relacionan con la actividad física de tiempo libre. A pesar de esto, se tiene la convicción de que la práctica de hoy condiciona en el alumnado la práctica del mañana, por consiguiente, resulta fundamental analizar las intenciones de práctica del alumnado, con el objetivo de conocer la forma de crear e inculcar hábitos de práctica en el tiempo libre. Por ello, el objetivo del presente trabajo ha sido validar, al idioma y al contexto español, el instrumento de medida de referencia de las intenciones de práctica deportiva en el tiempo libre: Intention to partake in leisure-time physical activity.

Como podemos apreciar, se obtienen correlaciones positivas entre la intención de realizar práctica física en el tiempo libre con la diversión en la práctica deportiva, coincidiendo con los resultados obtenidos por Standage et al. (2003) y corroborando además los datos de Ntoumanis $(2001,2002)$ al relacionarse con la motivación autodeterminada. Desde esta perspectiva, cuando un profesor diseña correctamente una clase, aumentan las posibilidades de que sus alumnos aprendan y se diviertan más. Coincidiendo con Cox et al. (2008) y a Deci y Ryan (2000), se puede afirmar que cuando el alumno se divierte, aumenta posiblemente su motivación intrínseca hacia la asignatura y las posibilidades de realizar actividad física fuera del colegio.

Tal como se aprecia en este trabajo, existe una relación positiva y significativa entre la importancia de la EF y la intención de práctica de actividad física en el tiempo libre. Como bien expresan Moreno y Hellín (2002), los alumnos de secundaria valoran positivamente la EF considerándola como una asignatura útil, manifestando además sus intereses hacia la práctica deportiva frente a otro tipo de actividades. Pero es interesante destacar la creencia de que el área de EF incide en el comportamiento de los alumnos hacia la práctica de actividad física en el tiempo libre. Sin embargo, Trost (2004) no encuentra evidencias que apoyen un vínculo consistente entre la asistencia a la asignatura de EF y la actividad física practicada fuera del colegio. Corroborando esta idea, Wilkin, Mallam y Metcalf (2006) concluyen que la asistencia del alumnado a EF explica menos del 1\% de la variación total de la actividad física que los alumnos realizan en su tiempo libre. Esto posiblemente se puede deber a que lo importante, para la cuestión que nos ocupa, no sea solo lo positivo de la cantidad de horas que asiste el alumnado a EF (Ardoy, et al., 2010), sino también la calidad de las clases, donde los alumnos si asisten motivados. Por ello, en este trabajo se obtiene una relación significativa entre las dos (destacando una mayor intención entre los chicos, sobre todo cuanto más jóvenes), y además se demuestra queexiste una relación positiva y significativa con la motivación intrínseca, posiblemente al contrario de lo que pasaría en los trabajos anteriores descritos, si se pudiera haber evaluado. Por tanto, siguiendo a Hagger y Chatzisarantis (2007), los datos de esta investigación corroboran que la existencia de motivación intrínseca hacia la EF puede conllevar a la práctica de actividad física en el tiempo de ocio, considerando por tanto a esta asignatura como importante.

Siguiendo a Taylor et al. (2010), cuando el profesor de EF adopta estrategias de motivación en sus clases, repercute en un mayor esfuerzo por parte del estudiante en esta área y en un aumento en las intenciones de práctica de actividad física en su tiempo libre. Asimismo, coincidimos con las aportaciones de Hagger y Chatzisarantis (2007), pues estos autores demuestran que las intenciones de los estudiantes de EF de hacer ejercicio físico durante las clases (posiblemente debido a una alta motivación), han demostrado ser un fuerte predictor de la conducta de realizar ejercicio físico en el tiempo libre. Este aspecto es entendible, pues los alumnos que asisten a clase de EF con intención de realizar mucha actividad física, seguramente es debido a que además, presentan una motivación intrínseca hacia la misma. Al respecto, se ha de destacar la importancia que el clima motivacional percibido y las necesidades psicológicas básicas tienen en la motivación intrínseca del alumnado (León, Domínguez, Núñez, Pérez \& Marín-Albo, 2011). De hecho, las motivaciones de los estudiantes de EF han sido asociadas con las intenciones de realizar actividad física en su tiempo libre, así como el esfuerzo realizado por ellos durante las clases de esta área (Hagger, Chatzisarantis, Barkoukis, Wang \& Baranowski, 2005; Ntoumanis, 2001; Standage, et al., 2003).

En la línea de los resultados obtenidos por Hagger, Cahtzisarantis, y Culverhouse (2003), Taylor et al. (2010) y Ullrich-French y Cox (2009), los datos del presente trabajo demuestran la relación positiva y significativa entre la motivación intrínseca y la intención de práctica de actividad física en el tiempo libre. Esto nos lleva a pensar, siguiendo a Chatzisarantis, Frederick, Biddle, Hagger y Smith (2007), que las intenciones de realizar una actividad física en el tiempo libre, aunque puede ser de forma obligada o voluntaria, en este caso queda claro que es de forma voluntaria.

Los datos muestran claramente los valores medios superiores de los chicos frente a las chicas en la intención de práctica física, denotando una mayor práctica de actividad física por parte de los chicos y corroborando los datos de otros autores (Granda, Montilla, Barbero, Mingorance \& Alemani, 2010; Trost, et al., 2002). Queda manifiesto, además, que los valores medios de intención de práctica física son mayores en los alumnos de 12 a 14 años que en el resto de las edades. Esto indica que la intención de realizar actividad física va disminuyendo conforme van aumentando en edad los alumnos, corroborando los resultados de otros trabajos que informan de la misma tendencia (Kim, et al., 2005).

Teniendo en cuenta todo lo anterior y los datos aportados y corroborados en otros trabajos de investigación de diversos autores, podemos concluir afirmando que la validación del Intention to partake in leisure-time physical activity en español contribuirá a reducir el vacío existente en investigación sobre las intenciones de práctica de actividad física durante el tiempo libre en adolescentes. Como se ha demostrado, se trata de un instrumento válido y fiable, por lo que sería de mayor trascendencia realizar nuevos estudios con diferentes muestras con este instrumento, para obtener nuevas evidencias de validez.

\section{Referencias}

Ajzen, I. (1991). The theory of planned behaviour. Organizational Behaviour and Human Decision Processes, 50, 179-211.

Ajzen, I., \& Fishbein, M. (1980). Understanding Attitudes and Predicting Social Behaviour. Englewood-Cliffs, NJ: Prentice-Hall.

Ajzen, I., \& Madden, T. (1986). Prediction of goal-directed behaviour: Attitudes, intentions and perceived behavioural control. Journal of Experimental Social Psychology, 22, 453-474.

Ardoy, D., Fernández-Rodríguez, J. M., Chillón, P., Artero, E., España-Romero, V., Jiménez-Pavón, D.,... Ortega, F. B. (2010). Educando para mejorar el estado de forma física, estudio EDUFIT: antecedentes, diseño, metodología y análisis del abandono/adhesión al estudio. Revista Española de Salud Pública, 84, 151-168.

Azjen, I. (1985). From intentions to actions: A Theory of Planned Behaviour. In J. Kuhl \& J. Beckman (Eds.), Action-Control: From cognition to behaviour (pp. 11-39). NY: Springer.

Baena-Extremera,A., Granero-Gallegos, A., Bracho-Amador, C., \& Pérez-Quero, F. J. (2012). Versión española del «Sport Satisfaction Instrument (SSI)» adaptado a la Educación Física. Revista de Psicodidáctica, 17(2), 375-365.

Bandura, A. (1977). Self-efficacy: Howard a unifying theory of behaviour change. Psychological Review, 84, 191-215.

Barrett, P. (2007). Structural equation modelling: Adjudging model fit. Personality and Individual Differences 42, 815-824.

Bentler, P. M. (2007). On tests and indices for evaluating structural models. Personality and Individual Differences 42, 825-829.

Bollen, K. A., \& Long, J. (1994). Testing structural equation models. Newbury Park, CA: Sage.

Brière, N., Vallerand, R., Blais, N., \& Pelletier, L. (1995). Développement et validation d'une mesure de motivation intrinsèque, extrinsèque et d'amotivation en contexte sportif: l'Échelle de motivation dans les sports (ÉMS). International 
Journal of Sport Psychology, 26, 465-489.

Brislin, R. W. (1986). The wording and translation of research instruments. In W. Lonner \& J. Berry (Eds.), Field methods in cross-cultural research (pp. 137164). Beverly Hills, CA: Sage.

Carretero-Dios, H., \& Pérez, C. (2007). Standards for the development and the review of instrumental studies: Considerations about test selection in psychological research. International Journal of Clinical and Health Psychology, 7, 863-882.

Chatzisarantis, N. L. D., Biddle, S. J. H., \& Meek, G. A. (1997). Aself-determination theory approach to the study of intentions and the intention-behaviour relationship in children's physical activity. British Journal of Health Psychology, 2, 343360 .

Chatzisarantis, N. L. D., Frederick, C. M., Biddle, S. J. H., Hagger, M. S., \& Smith, B. (2007). Influences of volitional and forced intentions on physical activity and effort within the theory of planned behavior. Journal of Sports Sciences, 25, 699-709.

Cox, A. E. Smith, A. L., \& Williams, L. (2008). Change in physical education motivation and physical activity behavior during middle school. The Journal of Adolescent Health, 43, 506-513.

Deci, E. L., \& Ryan, R. M. (1985). Intrinsic motivation and self determination in human behavior. New York: Plenum.

Deci, E. L., \& Ryan, R. M. (1991). A motivational approach to self: Integration in personality. In R. A. Dienstbier (Ed.), Nebraska symposium on motivation: Perspectives on Motivation (Vol. 38, pp. 237-288). Lincoln: University of Nebraska.

Deci, E. L., \& Ryan, R. M. (2000). The «what» and «why» of goal pursuits: Human needs and the self-determination of behavior. Psychological Inquiry, 11, 227-268.

Duda, J. L., \& Nicholls, J. G. (1992). Dimensions of achievement motivation in school work and sport. Journal of Educational Psychology, 84(3), 290-299.

Elosua, P., \& Zumbo, B. D. (2008). Coeficientes de fiabilidad para escalas de respuesta categórica ordenada. Psicothema, 20(4), 896-901.

Gómez-López, M., Granero-Gallegos, A., \& Baena-Extremera,A. (2010). Perceived barriers by university students in the practice of physical activities. Journal of Sports Science and Medicine, 9(3), 374-381.

Gómez-López, M., Granero-Gallegos, A., Baena-Extremera, A., \& Ruiz-Juan, F. (2011). The abandonment of an active lifestyle within university students: reasons for abandonment and expectations of re-engagement. Psychologica Belgica, 51(2), 155-175.

Goudas, M., Biddle, S., \& Fox, K. (1994). Perceived locus of causality, goa orientations, and perceived competence in school physical education classes. The British Journal of Educational Psychology, 64, 453-463.

Granda, J., Montilla, M., Barbero, J. C., Mingorance, A., \& Alemán, I. (2010). Frecuencia de práctica y motivos de participación/no participación en actividades físicas en función del género de escolares de 10-12 años de Melilla. Revista Internacional de Ciencias del Deporte, 21(6), 280-296.

Granero-Gallegos, A., Baena-Extremera, A., Pérez-Quero, J., Ortiz-Camacho, \& Bracho-Amador, C. (2012). Analysis of motivational profiles of satisfaction and importance of physical education in high school adolescents. Journal of Sports Science \& Medicine. In press.

Guzmán, J. F., Carratalá, E., \& Carratalá, V. (2005). Clima motivacional percibido y motivación deportiva: un estudio en deporte escolar. Comunicación presentada en el I Congreso de deporte en edad escolar «Propuestas para un nuevo modelo», Valencia, España.

Hagger, M. S. \& Chatzisarantis, N. L. D. (2007). The trans-contextual model of motivation. In M. S. Haggery N. L. D. Chatisarantis (Eds.), Intrinsic motivation and self-determination in exercise and sport (pp. 53-70). Leeds, UK: Human Kinetics.

Hagger, M. S., Chatzisarantis, N. L. D., Barkoukis, V., Wang, C. K. J., \& Baranowski, J. (2005). Perceive autonomy support in physical education promotes leisure-time physical activity intentions and behavior:A trans-contextual model. Journal of Educational Psychology, 95, 784-795.

Hagger, M. S., Chatzisarantis, N. L. D., Culverhouse, T., \& Biddle, S. J. H. (2003). The processes by which perceived autonomy support in physical education promotes leisure-time physical activity intentions and behavior: a trans-contextual model. Journal of Educational Psychology, 95, 784-795.

Hair, J. F., Black, W. C., Babin, B. J., \& Anderson, R. E. (2009). Multivariate Data Analysis (7th ed.). New York: Pearson Prentice Hall.

Hambleton, R. K. (2005). Issues, designs and technical guidelines for adapting tests into multiple languages and cultures. In R. K. Hambleton, P. F. Merenda, \& S. D. Spielberger (Eds.), Adapting educational and psychological tests for cross-cultural assessment (pp. 3-38). Mahwah, NJ: Lawrence Erlbaum Associates.

Hooper, D., Coughlan, J., \& Mullen, M. (2008). Structural Equation Modelling: Guidelines for Determining Model Fit. Electronic Journal of Business Research Methods, 6(1), 53-60.

Hu, L., \& Bentler, P. M. (1999). Cutoff criteria for fit indexes in covariance structure analysis: Conventional criteria versus new alternatives. Structural Equation Modelling, 6, 1-55.

Jöreskog, K. G. \& Sörbom, D. (1993). Structural equation modelling with the SIMPLIS command language. Chicago: Scientific Software International.
Kim, J., Must, A., Fitzmaurice, G. M., Gillman, M. W., Chomitz, V., Kramer, E.,... Peterson, K. E. (2005). Relationship of physical fitness to prevalence and incidence of overweight among schoolchildren. Obesity Research, 13(7), 12461254.

Kline, R. B. (2005), Principles and Practice of Structural Equation Modeling (2nd Edition ed.). New York: The Guilford Press.

León, J., Domínguez, E., Núñez, J. L., Pérez, A., \& Marín-Albo, J. (2011) Traducción y validación de la versión española de la Échelle de Satisfacción des Besoins Psychologiques en el contexto educativo. Anales de Psicología, 27(2), 405-411.

Lintunen, T., Valkonen, A., Leskinen, E., \& Biddle, S. J. H. (1999). Predicting physical activity intentions using a goal perspectives approach: A study of Finish youth. Scandinavian Journal of Medicine and Science in Sport, 9, 344352.

Markland, D. (2007). The golden rule is that there are no golden rules: A commentary on Paul Barrett's recommendations for reporting model fit in structural equation modelling. Personality and Individual Differences 42, 851-858.

Martens, M. P. \& Weber, S. N. (2002). Psychometric properties of the sport motivation scale: An evaluation with college varsity athletes from the U.S. Journal of Sport and Exercise Psychology, 24, 254-270.

Moreno, J. A. \& Hellín, P. (2002). ¿Es importante la Educación Física? Su valoración según la edad del alumno y el tipo de centro. Revista Internacional de Medicina y Ciencias de la Actividad Física y el Deporte, 2(8), 298-319.

Moreno, J.A., González-Cutre, D., \& Ruiz, L. M. (2009). Self-determined motivation and physical education importance. Human Movement, 10(1), 1-7.

Muñiz, J., \& Bartram, D. (2007). Improving international tests and testing. European Psychologist, 12, 206-219.

Ntoumanis N.(2002). Motivational clusters in a sample of British physical education classes. Psychology of Sport and Exercise, 3, 177-194.

Ntoumanis, N. (2001). Aself-determination approach to the understanding of motivation in physical education. British Journal of Educational Psychology, 71, 225242

Ntoumanis, N., Barkoukis, V., \& Thøgersen-Ntoumani, E.C. (2009). Developmental trajectories of motivation in physical education: course, demographic differences and antecedents. Journal of Educational Psychology, 101, 717-728.

Núñez, J. L., Martín-Albo, J., Navarro, J. G., \& González, V. M. (2006). Preliminary validation of a Spanish version of the Sport Motivation Scale. Perceptual and Motor Skills, 102, 919-930.

Nunnally, J. C., \& Bernstein, I. J. (1995).Teoría psicométrica. Madrid: McGrawHill.

Osterlind, S. J.(1989). Constructing Test Items. Londres: Kluwer Academic Publishers. Papaioannou, A. (2000). Attitudes, perceptions and behaviors (1) in the PE lesson, (2) in sport settings, (3) towards a healthy lifestyle, of individuals differing in gender, age, social class, religion and motor deficiency (Tech. Rep. No. 631). Thrace, Greece: Democritus University of Thrace.

Rhodes, R. E., Courneya, K. S., \& Jones, L. W. (2004). Personality and social cognitive influences on exercise behavior: Adding the activity trait to the theory of planned behavior. Psychology of Sport y Exercise, 5, 243-254.

Roman, B., Serra, L., Ribas, L., Pérez-Rodrigo, C., \& Aranceta, J. (2006). Actividad física en la población infantil y juvenil española en el tiempo libre. Estudio enKid (1998-2000). Apunts. Medicina de l'Esport, 41, 86-94.

Standage, M., Duda, J. L., \& Ntoumanis, N. (2003). A model of contextual motivation in physical education: Using constructs from self-determination and achievement goal theories to predict physical activity intentions. Journal of Educational Psychology, 95(1), 97-101.

Stevens, J. (1992). Applied multivariate statistics for the social sciences. Hillsdale, NJ: Lawrence Erlbaum.

Tabachnick, B. G., \& Fidell, L. S. (2007). Using Multivariate Statistics (5th ed.). New York: Allyn and Bacon.

Taylor, I. M., Ntoumanis, N., Standage, M., \& Spray, C. M. (2010). Motivational Predictors of Physical Education Students' Effort, Exercise Intentions, and Leisure-Time Physical Activity:AMultilevel Linear Growth Analysis. Journal of Sport and Exercise Psychology, 32, 99-120.

Trost, S. G., Pate, R. R., Sallis, J. F., Freedson, P. S., Taylor, W. C., Dowda, M., \& Sirard, J. (2002). Age and gender differences in objectively measured physical activity in youth. Medicine and Science in Sports and Exercise, 34, 350-355.

Trost, S. G. (2004). School physical education in the post-report era: an analysis from public health. Journal of Teaching in Physical Education, 23, 318-37.

Ullrich-French, S. \& Cox, A. (2009). Using cluster analysis to examine the combinations of motivation regulations of physical education students. Journal of Sport and Exercise Psychology, 31,358-379.

Wilkin, T. J., Mallam, K. M., \& Metcalf, B. S. (2006). Variation in physica activity lies with the child, not his environment: evidence for an «activity stat» in young children (Early Bird 16). International Journal of Obesity, 30, 10501055.

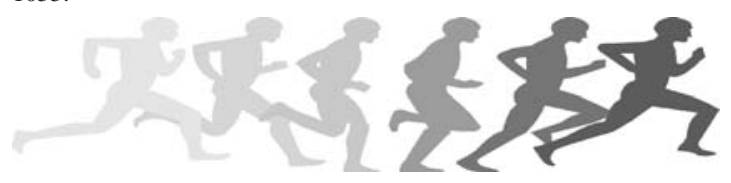

